\title{
Registro das Atividades Clínicas do Farmacêutico Hospitalar: Uma Revisão Integrativa
}

\author{
Émilin Dreher de Lima ${ }^{1}$, Carine Raquel Blatt ${ }^{2}$, Rita Catalina Aquino Caregnato ${ }^{3}$
}

\section{RESUMO}

Objetivo: Investigar como os farmacêuticos registram as intervenções clínicas no âmbito hospitalar. Método: Utilizou-se como método de pesquisa a revisão integrativa. Para realizar a busca dos artigos foram consultadas as fontes de informação Biblioteca Virtual em Saúde, Medical Literature Analysis and Retrieval System Online, Scientific Eletronic Library On-line, ScienceDirect e Web of Science, utilizando a combinação de termos e operadores booleanos "('pharmacist' OR 'pharmaceutical services' OR 'pharmacy service, hospital') AND ('hospitals') AND ('medical records' OR 'health records, personal' OR 'electronic health records' OR 'registries')". O nível de evidência foi avaliado segundo Melnyk e Fineout-Overholt (2011). Os artigos foram agrupados por proximidade de assunto. Resultados: 26 artigos preencheram os critérios de inclusão do estudo. A maioria dos estudos foi realizada em instituições dos Estados Unidos, publicada nos anos de 2014 e 2015. Apenas 31\% dos estudos reportam ao registro das atividades clínicas do farmacêutico em prontuário. Conclusões: Uma pequena parcela dos artigos sobre atividades clínicas do farmacêutico em hospital, deixa claro que o registro referente à prática de cuidado ao paciente foi realizado em prontuário. A maioria dos artigos relata o registro das atividades clínicas em um banco de dados distinto. O fato de o farmacêutico inserir informações referentes a suas atividades clínicas em bases de dados distinta do prontuário, pode facilitar mensuração de desfechos clínicos e econômicos. O registro da ação clínica no prontuário do paciente, contudo, não pode ser negligenciado, posto que é um direito do paciente e dever do profissional de saúde.

Palavras-chave: Farmacêuticos. Serviço de farmácia hospitalar. Registros eletrônicos de saúde. Registros médicos.

\section{THE REGISTRY OF HOSPITAL PHARMACEUTICALS' CLINICAL ACTIVITIES: AN INTEGRATIVE REVIEW}

ABSTRACT

Objective: To investigate how pharmacists record clinical interventions in hospitals. Method: The integrative review was used as a research method. The databases Biblioteca Virtual em Saúde, Medical Literature Analysis and Retrieval System Online, Scientific Eletronic Library Online, ScienceDirect and Web of Science have been referred to, using the combination of terms and boolean operators "('pharmacist' OR 'pharmaceutical services' OR 'pharmacy service, hospital') AND ('hospitals') AND ('medical records' OR 'health records, personal' OR 'electronic health records' OR 'registries')". The methodological quality of the studies was evaluated under criteria regarding level of evidence. The articles were grouped according to their subject relatedness. Results: 26 articles published between 2008 and 2018, in English, Spanish and Portuguese have been included. Most studies are originally form the USA, published in the years of 2014 and 2015. Only 31\% of the studies report registry of the pharmaceuticals' clinical activities on the medical records. Conclusions: A small portion of the articles on clinical activities of the pharmacist in a hospital makes it clear that the record regarding the practice of patient care was carried out in medical records. Most articles report the record of clinical activities in other database. The fact that the pharmacist inserts information related to his clinical activities in other databases can facilitate the measurement of clinical and economic outcomes. However, the record of clinical action in the patient's record cannot be neglected because it is the patient's right and duty the health professional. Keywords: Pharmacists. Pharmacy service hospital. Electronic health records. Medical records.

\section{RECEBIDO EM: 24/2/2020}

MODIFICAÇÕES SOLICITADAS EM: 6/4/2020

ACEITO EM: 7/4/2020

\footnotetext{
${ }^{1}$ Farmacêutica. Especialista em Atenção em Terapia Intensiva (UFCSPA/ISCMPA) e em Farmácia Hospitalar (SBRAFH). Mestranda em Ensino na Saúde pela Universidade Federal de Ciências da Saúde de Porto Alegre, Rio Grande de Sul, Brasil. http://lattes.cnpq.br/1440926300865518. https://orcid.org/00000002-8242-6130.emilin.d.I@hotmail.com

Farmacêutica. Doutora em Farmácia. Professora do Departamento de Farmacociências da Universidade Federal de Ciências da Saúde de Porto Alegre. Tutora da Residência Multiprofissional em Saúde (RMS) do Programa de Terapia Intensiva (UFCSPA/ISCMPA). Professora dos Programas de Pós-Graduação em Hepatologia, Mestrado Profissional em Enfermagem e Mestrado Profissional em Saúde da Família (UFCSPA). Rio Grande do Sul, Brasil. http://lattes.cnpq. br/4746842392238066. https://orcid.org/0000-0001-5935-1196. carineblatt@ufcspa.edu.br

${ }^{3}$ Enfermeira. Doutora em Educação. Professora do Departamento de Enfermagem da Universidade Federal de Ciências da Saúde de Porto Alegre. Professora permanente dos Programas de Pós-Graduação Mestrado Profissional em Ensino na Saúde e Mestrado Profissional em Enfermagem (UFCSPA). Tutora da Residência Multiprofissional em Saúde (RMS) do Programa de Terapia Intensiva (UFCSPA/ISCMPA). Rio Grande de Sul, Brasil. http://lattes.cnpq. br/5672094236990422 https://orcid.org/0000-0001-7929-7676. ritac@ufcspa.edu.br
} 


\section{INTRODUÇÃO}

A Farmácia Clínica é uma área de atuação farmacêutica relativamente nova, com origem na década de 60 nos Estados Unidos e, desde então, o conceito vem difundindo-se e a prática se consolidando (MENEZES, 2000). Na prática da Farmácia Clínica, a proposta de otimização da farmacoterapia e uso racional de medicamentos tem a finalidade de melhorar a qualidade de vida dos pacientes, aliada à redução de custos. Essa área de atuação aproxima o farmacêutico do paciente e da equipe de saúde (MENEZES, 2000; FERRACINI et al., 2011).

No Brasil, o movimento da Farmácia Clínica teve sua origem reportada em Natal (RN), no hospital universitário da Universidade Federal do Rio Grande do Norte, no final da década de 70 (CUNHA; BRANDÃO, 2010). Apenas em 2013, no entanto, o Conselho Federal de Farmácia (CFF) publicou a resolução no 585 , que regulamenta as atribuições clínicas do farmacêutico (BRASIL, 2013). Dentre as atribuições dispostas nesse documento, encontra-se a descrição da responsabilidade do farmacêutico em relação ao registro de suas intervenções no prontuário do paciente por meio da evolução farmacêutica (BRASIL, 2013).

Previamente à publicação da Resolução no 585, em 2008 o CFF já havia regulamentado o registro das intervenções farmacêuticas em prontuário do paciente, regulamentação esta que foi revisada em 2011 (BRASIL, 2008, 2011). Mesmo, contudo, após dez anos da regulamentação do registro farmacêutico no prontuário do paciente, observa-se que esta prática ainda não está consolidada entre os profissionais. Recente estudo realizado em um hospital na Região Sul do Brasil, mostrou que os farmacêuticos reconhecem a importância do registro das intervenções farmacêuticas no prontuário do paciente, entretanto frequentemente não o fazem (LIMA et al., 2017).

Esse cenário também é evidenciado em outros países (PULLINGER; FRANKLIN, 2010; FRONTINI; MIHARIJA-GALA; SYKORA, 2013; ALSULTAN et al., 2013). Estudo conduzido em um hospital de Londres (UK) destacou que $74 \%$ dos farmacêuticos entrevistados não registram suas intervenções em prontuário (PULLINGER; FRANKLIN, 2010). Uma análise realizada pela European Association of Hospital Pharmacists (EAHP) mostrou que, em média, apenas $14,7 \%$ das intervenções farmacêuticas são registradas em prontuário (FRONTINI; MIHARIJA-GALA; SYKORA, 2013). Estudo realizado com coordenadores de farmácias de hospitais da região de Riade, capital da Arábia Saudita, constatou que apenas $24 \%$ das instituições deter- minam a obrigatoriedade do registro das atividades clínicas do farmacêutico no prontuário do paciente (ALSULTAN et al., 2013).

Em virtude ao que foi exposto, e compreendendo que o prontuário do paciente é documento oficial de registro das atividades clínicas, garantia da continuidade do cuidado em ambiente hospitalar e ferramenta indispensável de comunicação entre a equipe de saúde, o objetivo do presente estudo é investigar como os farmacêuticos registram as intervenções clínicas no âmbito hospitalar.

\section{MÉTODO}

Trata-se de uma revisão integrativa que cumpriu as seis etapas de pesquisa: (1) identificação do tema e seleção da questão de pesquisa; (2) estabelecimento dos critérios de elegibilidade; (3) identificação dos estudos nas bases científicas; (4) avaliação dos estudos selecionados e análise crítica; (5) categorização dos estudos; e (6) avaliação e interpretação dos resultados com apresentação dos dados na estrutura da revisão integrativa (BOTELHO; CUNHA; MACEDO, 2011). A questão norteadora do estudo foi: "Como são realizados os registros das intervenções farmacêuticas em pacientes internados em hospital?"

Os critérios de inclusão estabelecidos foram: artigos científicos publicados nos idiomas português, espanhol ou inglês, que apresentem relatos de intervenções clínicas do farmacêutico no âmbito hospitalar, publicados no período de 2008 a 2018. O ano de 2008 foi determinado para início da pesquisa por se tratar do marco regulatório do registro farmacêutico em prontuário do paciente no Brasil, com a resolução no 476 (BRASIL, 2008), que regulamentou o registro, a guarda e o manuseio de informações resultantes da prática da assistência farmacêutica nos serviços de saúde, atualmente revogada pela resolução no 555 (BRASIL,2011). Os critérios de exclusão foram: artigos que não reportavam o documento utilizado para o registro da intervenção farmacêutica e estudos duplicados.

Foram definidas as seguintes fontes de informação para busca: Biblioteca Virtual em Saúde (BVS-Bireme), Medical Literature Analysis and Retrieval System Online (MEDLINE/PubMed), Scientific Eletronic Library Online (Scielo), ScienceDirect e Web of Science. A combinação de termos e operadores booleanos utilizados nesta pesquisa foi: ("pharmacist" OR "pharmaceutical services" OR "pharmacy service, hospital") AND ("hospitals") AND ("medical records" OR "health records, personal" $O R$ "electronic health records" OR "registries"). A síntese das etapas de pesquisa é apresentada na Figura 1. 
Figura 1 - Representação das etapas da revisão integrativa para seleção de estudos sobre registro das atividades clínicas do farmacêutico no ambiente hospitalar

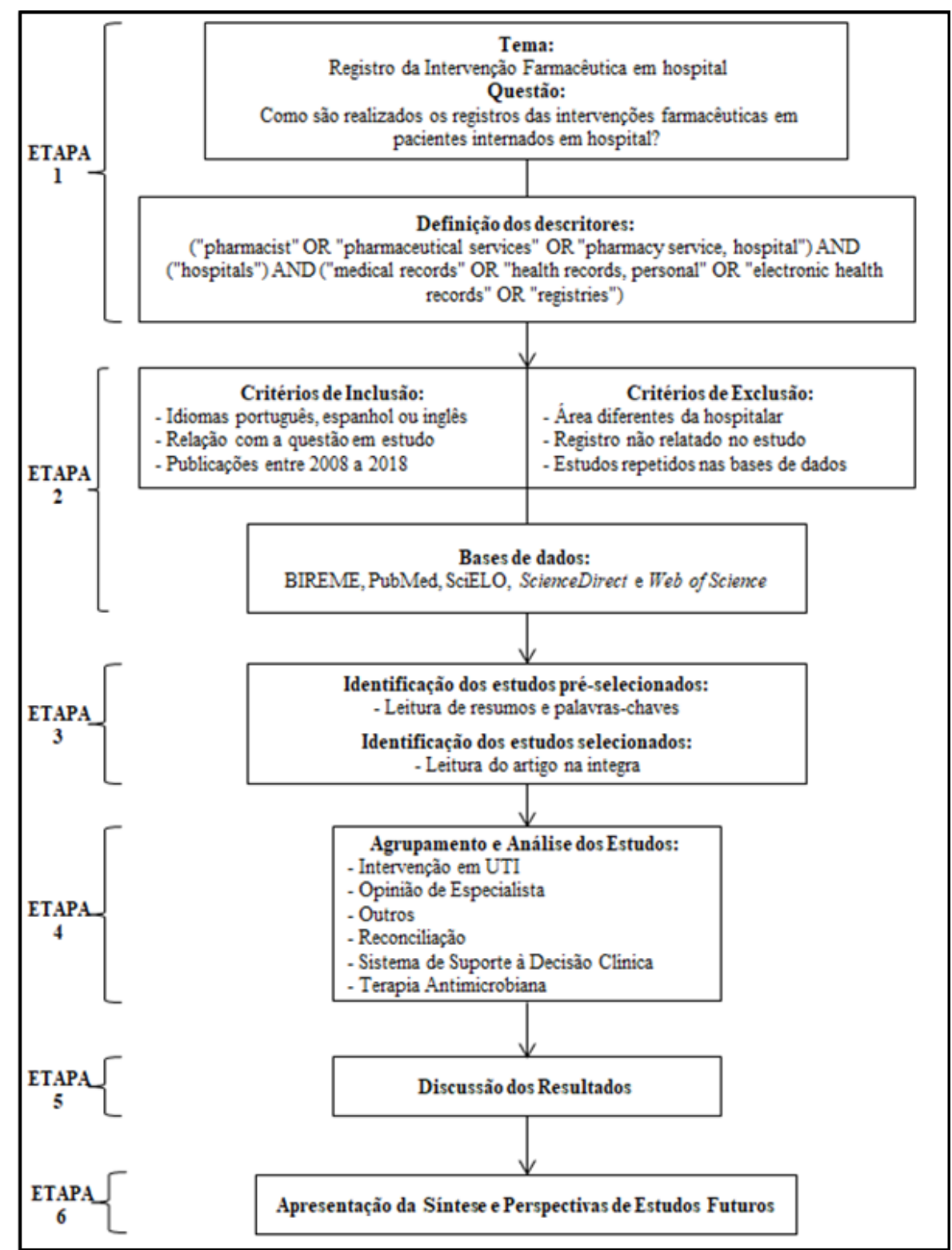

Fonte: As autoras (2018).

Os estudos selecionados foram classificados em sete níveis de evidência: 1) revisão sistemática ou metanálise; 2) ensaios clínicos randomizados; 3) ensaios clínicos sem randomização; 4) caso-controle e de corte; 5) evidência de revisões sistemáticas de estudos descritivos e qualitativos; 6 ) estudos descritivos ou qualitativos simples; 7) opinião de especialistas e/ ou relatórios de comitês de especialistas (MELNYK; FINEOUT-OVERHOLT, 2011).

Dados de interesse foram coletados das publicações selecionadas por meio de um instrumento com as seguintes variáveis: título, autor, ano, país, tipo de estudo, forma de registro das atividades clínicas, ob- jetivos, resultados e conclusões de cada estudo. A forma de registro foi classificada em prontuário como "sim" ou "não informado" (quando o estudo trazia em seu texto a descrição de outro documento para registro das intervenções, porém não relatava que a intervenção não havia sido registrada em prontuário). Os estudos foram agrupados em seis categorias, sendo: terapia antimicrobiana; conciliação medicamentosa; sistema de suporte à decisão clínica, opinião de especialista, unidade de terapia intensiva (UTI); e outros. Para facilitar a identificação os estudos receberam um código A1 a A26. Os dados principais foram apresentados na seção de resultados. 


\section{RESULTADOS}

O retorno da busca identificou um total de 299 artigos. Desses, 39 atendiam os critérios de inclusão do estudo, porém 13 estavam em duplicata, resultando um total de 26 artigos inclusos nesta revisão (Tabela 1).

Tabela 1 - Síntese por base de dados da busca de estudos sobre o registro das atividades clínicas do farmacêutico no ambiente hospitalar

\begin{tabular}{|c|c|c|c|c|}
\hline Base de Dados & $\begin{array}{l}\text { Retorno da } \\
\text { Busca (n) }\end{array}$ & $\begin{array}{c}\text { Artigos } \\
\text { Pertinentes } \\
\text { (n) }\end{array}$ & $\begin{array}{l}\text { Artigos } \\
\text { Duplicados } \\
\text { (n) }\end{array}$ & $\begin{array}{l}\text { Artigos } \\
\text { Incluídos } \\
\text { (n) }\end{array}$ \\
\hline BIREME & 118 & 17 & 0 & 17 \\
\hline Pubmed & 113 & 12 & 11 & 1 \\
\hline SciELO & 1 & 0 & 0 & 0 \\
\hline ScienceDirect & 40 & 7 & 1 & 6 \\
\hline Web of Science & 27 & 3 & 1 & 2 \\
\hline Total & 299 & 39 & 13 & 26 \\
\hline
\end{tabular}

Fonte: As autoras (2018).
Os países de origem dos estudos incluídos nesta revisão foram: Estados Unidos (23\%); Austrália (11\%); China (11\%); Espanha (11\%); Bélgica (8\%); Irlanda (8\%); Inglaterra (8\%); África do Sul (4\%); Arábia Saudita (4\%); Brasil (4\%); Dinamarca (4\%); e Israel (4\%). A distribuição dos artigos por ano de publicação é: 2008 (4\%); 2009 (4\%); 2010 (11\%); 2011 (15\%); 2012 (8\%); 2013 (8\%); 2014 (19\%); 2015 (19\%); 2016 (4\%); 2017 (4\%); e 2018 (4\%).

Quanto ao tipo de estudo, constatou-se: prospectivo (46\%); retrospectivo (27\%); longitudinal (19\%); e transversal (8\%). Em relação ao nível de evidência (NE) observou-se: 1 estudo com NE II (4\%); 3 com NE IV (11\%); 21 com NE VI (77\%); e 2 com NE VII (8\%). O Quadro 1 apresenta a síntese dos estudos incluídos nesta revisão integrativa. Predominaram os estudos de intervenção e o nível de evidência NE VI, conforme pode ser visualizado no Quadro 1. 
Quadro 1 - Síntese dos estudos incluídos na revisão integrativa sobre o registro das atividades clínicas do farmacêutico no ambiente hospitalar

\begin{tabular}{|c|c|c|c|c|c|c|c|}
\hline Grupo & Artigo & Título & País & Tipo de Estudo & Ano & $\begin{array}{c}\text { Registro em } \\
\text { Prontuário }\end{array}$ & $\begin{array}{l}\text { Nível de } \\
\text { Evidência }\end{array}$ \\
\hline \multirow{8}{*}{ 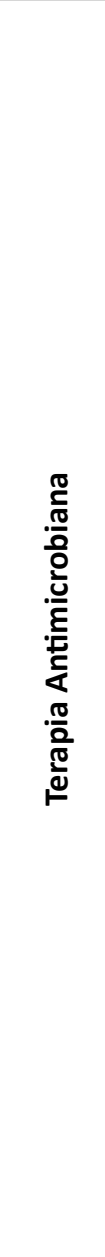 } & $\mathrm{A} 1$ & \begin{tabular}{|c|} 
Impact of antimicrobial stewardship \\
program on vancomycin use in a pediatric \\
teaching hospital*
\end{tabular} & $\begin{array}{l}\text { Estados } \\
\text { Unidos }\end{array}$ & Prospectivo & 2010 & $\begin{array}{l}\text { Não } \\
\text { informado }\end{array}$ & VI \\
\hline & $\mathrm{A} 2$ & $\begin{array}{l}\text { Implementing a pharmacist-led } \\
\text { sequential antimicrobial therapy strategy: } \\
\text { a controlled before-and-after study* }\end{array}$ & Irlanda & Prospectivo & 2011 & $\begin{array}{l}\text { Não } \\
\text { informado }\end{array}$ & IV \\
\hline & A3 & $\begin{array}{c}\text { Implementation of intravenous to oral } \\
\text { antibiotic switch therapy guidelines in the } \\
\text { general medical wards of a tertiary-level } \\
\text { hospital in South Africa* }\end{array}$ & $\begin{array}{l}\text { África do } \\
\text { Sul }\end{array}$ & Longitudinal & 2011 & $\begin{array}{c}\text { Não } \\
\text { informado }\end{array}$ & VI \\
\hline & A4 & $\begin{array}{c}\text { Implementation of an antimicrobial } \\
\text { stewardship program on the medical- } \\
\text { surgical service of a } 100-\text {-bed community } \\
\text { hospital* }\end{array}$ & $\begin{array}{l}\text { Estados } \\
\text { Unidos }\end{array}$ & Prospectivo & 2012 & $\begin{array}{l}\text { Não } \\
\text { informado }\end{array}$ & VI \\
\hline & A5 & $\begin{array}{l}\text { Impact of an antimicrobial stewardship } \\
\text { program on patients with acute bacterial } \\
\text { skin and skin structure infections* }\end{array}$ & $\begin{array}{l}\text { Estados } \\
\text { Unidos }\end{array}$ & Retrospectivo & 2014 & Sim & VI \\
\hline & A6 & $\begin{array}{c}\text { Pharmacist interventions for prophylactic } \\
\text { antibiotic use in urological inpatients } \\
\text { undergoing clean or clean contaminated } \\
\text { operations in a Chinese Hospital* }\end{array}$ & China & Longitudinal & 2014 & $\begin{array}{l}\text { Não } \\
\text { informado }\end{array}$ & VI \\
\hline & A7 & $\begin{array}{c}\text { Effects of a bundled Antimicrobial } \\
\text { Stewardship Program on mortality: a } \\
\text { cohort study* }\end{array}$ & Brasil & Retrospectivo & 2015 & Sim & IV \\
\hline & A8 & $\begin{array}{c}\text { Optimizing prophylactic antibiotic } \\
\text { practice for cardiothoracic surgery by } \\
\text { pharmacists' effects* }\end{array}$ & China & Longitudinal & 2016 & $\begin{array}{l}\text { Não } \\
\text { informado }\end{array}$ & VI \\
\hline \multirow{3}{*}{ 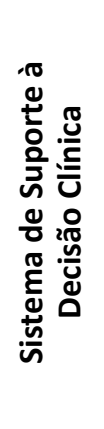 } & A9 & $\begin{array}{c}\text { Adopting real-time surveillance } \\
\text { dashboards as a component of an } \\
\text { enterprisewide medication safety } \\
\text { strategy* }\end{array}$ & $\begin{array}{l}\text { Estados } \\
\text { Unidos }\end{array}$ & Prospectivo & 2011 & Sim & VI \\
\hline & A10 & $\begin{array}{c}\text { Sistemas de soporte a la toma de } \\
\text { decisiones clínicas en insuficiência renal* }\end{array}$ & Espanha & Longitudinal & 2014 & $\begin{array}{l}\text { Não } \\
\text { informado }\end{array}$ & VI \\
\hline & A11 & $\begin{array}{l}\text { The impact of a structured pharmacist } \\
\text { intervention on the appropriateness of } \\
\text { prescribing in older hospitalized patients* }\end{array}$ & Irlanda & Retrospectivo & 2014 & $\begin{array}{c}\text { Não } \\
\text { informado }\end{array}$ & VI \\
\hline
\end{tabular}




\begin{tabular}{|c|c|c|c|c|c|c|c|}
\hline \multirow{6}{*}{ 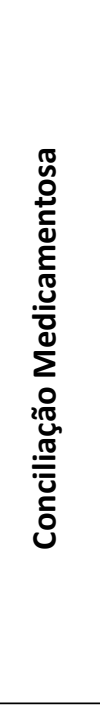 } & A12 & $\begin{array}{c}\text { Estudio prospectivo de conciliación } \\
\text { de medicación en pacientes de } \\
\text { traumatologia* }\end{array}$ & Espanha & Prospectivo & 2008 & $\begin{array}{c}\text { Não } \\
\text { informado }\end{array}$ & $\mathrm{VI}$ \\
\hline & A13 & $\begin{array}{l}\text { Pharmacists' medication reconciliation- } \\
\text { related clinical interventions in a } \\
\text { children's hospital* }\end{array}$ & $\begin{array}{l}\text { Estados } \\
\text { Unidos }\end{array}$ & Prospectivo & 2009 & Sim & VI \\
\hline & A14 & $\begin{array}{l}\text { Added value of pharmacist-acquired drug } \\
\text { histories in an orthopaedic ward* }\end{array}$ & Bélgica & Prospectivo & 2011 & Sim & $\mathrm{VI}$ \\
\hline & A15 & $\begin{array}{c}\text { An audit of the pharmacological } \\
\text { management of ischaemic stroke patients } \\
\text { in a metropolitan Australian hospital* }\end{array}$ & Austrália & Retrospectivo & 2015 & Sim & IV \\
\hline & A16 & $\begin{array}{c}\text { Adecuación de la historia } \\
\text { farmacoterapéutica y errores de } \\
\text { conciliación en un servicio de urgências* }\end{array}$ & Espanha & Prospectivo & 2015 & $\begin{array}{c}\text { Não } \\
\text { informado }\end{array}$ & $\mathrm{VI}$ \\
\hline & A17 & $\begin{array}{l}\text { Medication review by a clinical } \\
\text { pharmacist at the transfer point from ICU } \\
\text { to ward: a randomized controlled trial* }\end{array}$ & Bélgica & Prospectivo & 2015 & $\begin{array}{c}\text { Não } \\
\text { informado }\end{array}$ & II \\
\hline \multirow{5}{*}{ 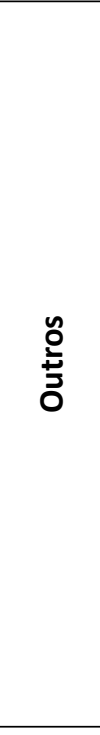 } & A18 & $\begin{array}{c}\text { Clinical pharmacy workload in medical } \\
\text { and surgical patients: effect of patient } \\
\text { partition, disease complexity and Major } \\
\text { Disease Category* }\end{array}$ & Austrália & Prospectivo & 2010 & $\begin{array}{c}\text { Não } \\
\text { informado }\end{array}$ & $\mathrm{VI}$ \\
\hline & A19 & $\begin{array}{l}\text { Electronic health record identification } \\
\text { of nephrotoxin exposure and associated } \\
\text { acute kidney injury* }\end{array}$ & $\begin{array}{l}\text { Estados } \\
\text { Unidos }\end{array}$ & Prospectivo & 2013 & $\begin{array}{c}\text { Não } \\
\text { informado }\end{array}$ & $\mathrm{VI}$ \\
\hline & A20 & $\begin{array}{c}\text { Characterization of drug-related problems } \\
\text { identified by clinical pharmacy staff at } \\
\text { Danish hospitals* }\end{array}$ & Dinamarca & Retrospectivo & 2014 & $\begin{array}{c}\text { Não } \\
\text { informado }\end{array}$ & $\mathrm{VI}$ \\
\hline & A21 & $\begin{array}{l}\text { Impact of clinical pharmacist } \\
\text { interventions on inappropriate } \\
\text { prophylactic acid suppressant use } \\
\text { in hepatobiliary surgical patients } \\
\text { undergoing elective operations* }\end{array}$ & China & Longitudinal & 2017 & $\begin{array}{c}\text { Não } \\
\text { informado }\end{array}$ & $\mathrm{VI}$ \\
\hline & A22 & $\begin{array}{l}\text { Antidiabetics' usage in type } 2 \text { diabetes } \\
\text { mellitus: Are prescribing guidelines } \\
\text { adhered to? A single centre study* }\end{array}$ & Austrália & Retrospectivo & 2018 & Sim & $\mathrm{VI}$ \\
\hline \multirow{2}{*}{ 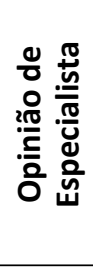 } & A23 & $\begin{array}{c}\text { Pharmacists' documentation in patients' } \\
\text { hospital health records: issues and } \\
\text { educational implications* }\end{array}$ & Inglaterra & Transversal & 2010 & $\begin{array}{c}\text { Não } \\
\text { informado }\end{array}$ & VII \\
\hline & A24 & $\begin{array}{l}\text { Hospital pharmacy practice in Saudi } \\
\text { Arabia: Drug monitoring and patient } \\
\text { education in the Riyadh region* }\end{array}$ & $\begin{array}{l}\text { Arábia } \\
\text { Saudita }\end{array}$ & Transversal & 2013 & Sim & VII \\
\hline \multirow{2}{*}{ 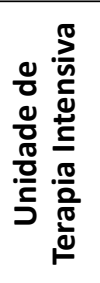 } & A25 & $\begin{array}{c}\text { Pharmacist proactive medication } \\
\text { recommendations using electronic } \\
\text { documentation in a UK general critical } \\
\text { care unit* }\end{array}$ & Inglaterra & Retrospectivo & 2012 & $\begin{array}{c}\text { Não } \\
\text { informado }\end{array}$ & $\mathrm{VI}$ \\
\hline & A26 & $\begin{array}{c}\text { Pharmacist remote review of medication } \\
\text { prescriptions for appropriateness in } \\
\text { pediatric intensive care unit* }\end{array}$ & Israel & Prospectivo & 2015 & $\begin{array}{l}\text { Não } \\
\text { informado }\end{array}$ & $\mathrm{VI}$ \\
\hline
\end{tabular}

*Referências: A1 (DI PENTIMA; CHAN, 2010); A2 (DUNN et al., 2011); A3 (VAN NIEKERK; VENTER; BOSCHMANS, 2011); A4 (STOREY et al., 2012); A5 (PASQUALE et al., 2014); A6 (ZHANG et al., 2014); A7 (OKUMURA; SILVA; VERONEZE, 2015); A8 (ZHOU et al., 2016); A9 (WAITMAN et al., 2011); A10 (BERNABÉ et al., 2014); A11 (O'SULLIVAN et al., 2014; A12 (MORIEL et al., 2008); A13 (GARDNER; GRANER, 2009); A14 (QUENNERY et al., 2011 ); A15 (KHALIL; LI; HUA, 2015); A16 (ANDRÉS-LÁZARO et al., 2015); A17 (HESELMANS et al., 2015); A18 (STUCHBERY et al., 2010); A19 (GOLDSTEIN et al., 2013 ); A20 (KJELDSEN et al., 2014); A21 (LUO et al., 2017); A22 (KHALIL et al., 2018); A23 (PULLINGER; FRANKLIN, 2010); A24 (ALSULTAN et al., 2013); A25 (BOURNE; CHOO, 2012 ); A26 (LAZARYAN et al., 2016). 
A distribuição dos artigos por tema abordado permitiu o agrupamento em seis grupos, a saber: terapia antimicrobiana (31\%); conciliação medicamentosa (23\%); sistema de suporte à decisão clínica (11\%); opinião de especialista (8\%); unidade de terapia intensiva (UTI) (8\%); e outros (19\%). Constatou-se $31 \%(n=8)$ dos artigos apontam o registro da atividade clínica em prontuário, e os outros $69 \%(n=18)$ indicam o registro em outro documento. Dentre os artigos que relatavam o registro da atividade clínica em prontuário, $75 \%$ $(n=6)$ mostravam que o registro também foi realizado em outro banco de dados acessível apenas ao farmacêutico.

\section{DISCUSSÃO}

O registro das atividades clínicas do farmacêutico é um tema que ainda traz certa apreensão para a classe profissional em âmbito nacional e mundial (MENEZES, 2000; CUNHA; BRANDÃO, 2010; FOX; PEDERSEN; GUMPPER, 2015). O farmacêutico tem experiência na manutenção de diversos registros, mas muitos não têm experiência em documentar as atividades de atendimento ao paciente (APHA, 2007).

Observou-se nos resultados que os farmacêuticos registram suas atividades clínicas em banco de dados com frequência maior do que o fazem em prontuário. Esse resultado pode ser explicado pelo desafio de elaboração do registro da atividade de forma concisa e inteligível a todos os profissionais de saúde, pelo fato de a atividade clínica do farmacêutico estar vinculada à otimização da farmacoterapia e ao uso racional de medicamentos, o que, por vezes, perpassa pela identificação de necessidade de ajuste na prescrição e pode abordar situações de falta de segurança no uso dos medicamentos (BRASIL, 2011; PULLINGER; FRANKLIN, 2010; MACKINNON; MACKINNON, 2011).

Além disso, a inexperiência e o desconhecimento de métodos de registro, a sobrecarga de atividades e a indisponibilidade de tempo destes profissionais, também são fatores limitantes para a realização da atividade (LIMA et al., 2017). Por outro lado, com o aumento da necessidade de registro das informações e do olhar interprofissional para a segurança do paciente, este registro pode contribuir para a identificação precoce e manejo adequado do erro de medicação (PREBIANCHI, 2017; PAVÃO et al., 2011).

Além disso, o farmacêutico usualmente costuma registrar apenas quando realiza alguma intervenção, contudo o fato de avaliar a prescrição ou o estado de saúde do paciente, ou monitorar os níveis séricos dos medicamentos, verificar a presença ou não de reação adversa a medicamento, interações, incompatibilidades, também deve ser registrado (BRASIL, 2011, 2013; LIMA et al., 2017). Ou seja, toda a atividade clínica deve ser registrada, independentemente de que esta resulte ou não em uma intervenção (BRASIL, 2011, 2013).

\section{Terapia Antimicrobiana}

O manejo da terapia antimicrobiana junto a equipe médica é um campo crescente de atuação para o farmacêutico clínico (PARENTE; MORTON, 2018; WHITNEY et al., 2019). Este campo vai ao encontro da ação do farmacêutico de promover o uso racional de medicamentos, visando a adequação do medicamento à condição clínica do paciente, ao sítio da infecção, às características do agente infeccioso e às possibilidades de via para administração dos medicamentos (PARENTE; MORTON, 2018; MORGAN et al., 2018). Além de promover o uso dos antimicrobianos de forma mais segura e efetiva, a intervenção do farmacêutico mostra-se eficiente na redução de custos (WHITNEY et al., 2019; SCHNEIDER; PEDERSEN; SCHECKELHOFF, 2018).

Observou-se entre os estudos inclusos neste grupo dois artigos (A5 e A7) que, em sua redação, descrevem o registro da intervenção farmacêutica no prontuário do paciente (PASQUALE et al., 2014; OKUMURA; SILVA; VERONEZE, 2015). Além disso, os relatos do manejo da terapia antimicrobiana ocorreram em diferentes momentos e/ou grupos específicos de pacientes. Os artigos A8 e A6 apresentaram a atuação do farmacêutico no apoio ao uso de antimicrobianos nos momentos pré e/ou pós-operatório, em distintos cenários (ZHANG et al., 2014; ZHOU et al., 2016).

No artigo $A 7$ os farmacêuticos clínicos ou o farmacêutico dedicado ao time de manejo antimicrobiano, faziam buscas de possibilidades de melhoria na terapia cruzando o resultado de culturas, prescrição e registros em prontuário (OKUMURA; SILVA; VERONEZE, 2015). A substituição por via de administração menos invasiva (via endovenosa para via oral) também foi apresentada como alvo para ação do farmacêutico (A3 e A2) (DUNN et al., 2011; VAN NIEKERK; VENTER; BOSCHMANS, 2011). A atuação em relação a um antimicrobiano específico foi apresentada no artigo A1 (DI PENTIMA; CHAN, 2010). O tempo inicial do tratamento sistêmico foi gatilho para as ações no artigo A4 (STOREY et al., 2012). O artigo A5 apresenta resultado de intervenções realizadas a pacientes a partir da identificação de uma Classificação Internacional de Doenças (CID) específica (PASQUALE et al., 2014). 


\section{Sistema de Suporte à Decisão Clínica}

Os sistemas de suporte à decisão clínica estão sendo empregados como estratégia para auxiliar os profissionais farmacêuticos e médicos na análise, escolha e otimização da terapia medicamentosa $(\mathrm{SCH}-$ NEIDER; PEDERSEN; SCHECKELHOFF, 2018; ENGLISH; ANKEM; ENGLISH, 2017). Dentre os artigos integrantes deste grupo, verificou-se um que descreveu o registro da intervenção farmacêutica no prontuário do paciente.

$\mathrm{O}$ artigo $\mathrm{A} 10$ relata o uso de sistemas de suporte à decisão clínica no ajuste da dosagem dos medicamentos de acordo com a função renal do paciente, calculada a partir dos valores de filtração glomerular (BERNABÉ et al., 2014). O emprego de sistemas de suporte à decisão clínica no artigo A11 é dado como instrumento aliado à reconciliação medicamentosa por farmacêutico, indicando discrepância entre os medicamentos de uso prévio e prescritos, e alertando quanto a problemas relacionados à utilização concomitante de alguns medicamentos, ou o uso não indicado de algum medicamento para determinada condição clínica (O'SULLIVAN et al., 2014). Já o artigo A9 relata o uso de sistemas de suporte à decisão clínica para identificação da prescrição de medicamentos de alto risco, que são o foco da intervenção farmacêutica (WAITMAN et al., 2011).

\section{Conciliação Medicamentosa}

A obtenção de um histórico acurado de medicamentos de uso pré, intra e pós-internação em ambiente hospitalar, é uma valiosa ferramenta de prevenção a problemas relacionados ao uso de medicamentos. A conciliação medicamentosa pode ser especialmente útil quando aplicada a pacientes que utilizam esquemas terapêuticos complexos, por exemplo, pacientes em uso de cinco medicamentos ou mais (polifarmácia) e pacientes transplantados. No ato da conciliação medicamentosa é possível identificar divergências, otimizar a terapia, avaliar a adesão do paciente ao tratamento e verificar a adequação da prescrição a protocolos institucionais, nacionais e/ou internacionais (KHALIL; LI; HUA, 2015; LOMBARDI et al., 2016; MONGARET et al., 2018). Neste grupo foi identificado o maior percentual (50\%) de estudos que informaram no corpo do artigo o registro da intervenção farmacêutica no prontuário do paciente (A13, A14 e A15) (GARDNER; GRANER, 2009; QUENNERY et al., 2011; KHALIL; LI; HUA, 2015).

Observa-se neste grupo artigos relacionados à análise de dados referente à intervenção farmacêutica em condições de saúde específicas (A15 - acidente vascular cerebral isquêmico) (KHALIL; LI; HUA, 2015), com populações específicas (A13 - paciente pediátrico; A12 - pacientes com 65 anos ou mais admitidos pelo serviço de traumatologia) (MORIEL et al., 2008; GARDNER; GRANER, 2009), em unidade hospitalar específica (A16 - serviço de emergência e A14 - serviço de ortopedia) (QUENNERY et al., 2011; ANDRÉS-LÁZARO et al., 2015) e na transição de pacientes entre unidades de um hospital (A17 - pacientes transferidos de unidade de terapia intensiva para enfermaria) (HESELMANS et al., 2015).

\section{Opinião de Especialista}

Neste grupo foram inclusos dois estudos (PULLINGER; FRANKLIN, 2010; ALSULTAN et al., 2013). $\mathrm{O}$ primeiro foi realizado em um hospital de Londres, no qual 40 farmacêuticos participaram da pesquisa (A23) (PULLINGER; FRANKLIN, 2010). O estudo demonstrou que os farmacêuticos tinham preferência em registrar suas intervenções de forma verbal e por meio de mensagens temporárias no sistema informatizado, sem manter um registro permanente no prontuário do paciente (PULLINGER; FRANKLIN, 2010). O segundo estudo foi realizado com diretores de farmácias hospitalares de uma determinada região da Arábia Saudita (A24). Este mostrou que em apenas 52\% dos hospitais da região os farmacêuticos realizavam o registro das intervenções em prontuário, conforme informação dos gestores do serviço (ALSULTAN et al., 2013).

\section{Unidade de Terapia Intensiva}

A atuação do farmacêutico clínico é especialmente importante em unidades que fazem o atendimento de paciente crítico, como emergência e unidade de terapia intensiva. Nestas áreas, o farmacêutico, além de fazer a revisão completa da farmacoterapia, com o intuito de otimizar e adequar a terapia medicamentosa às necessidades específicas de cada paciente, atua favorecendo a adesão às diretrizes de prática clínica, realizando a monitorização terapêutica de medicamentos, além de desempenhar importante papel na educação continuada da equipe multiprofissional e auxílio à introdução de novas rotinas institucionais (JURADO; STEELMAN, 2013).

Nos estudos apresentados pelos artigos A25 e 26, o farmacêutico clínico realizou revisão completa da prescrição de medicamentos do paciente internado em unidade de terapia intensiva, a fim de identificar melhorias e adequações na terapia medicamentosa (BOURNE; CHOO, 2012; LAZARYAN et al., 2016). 
Ambos os artigos informam que as intervenções clínicas do farmacêutico foram registradas em banco de dados e não mencionam o registro em prontuário do paciente.

\section{Outros}

O grupo "outros" é composto por seis artigos. Destes seis artigos, apenas um (A22) apresenta em seu texto a informação da busca do registro da ação clínica do farmacêutico no prontuário do paciente (KHALIL et al., 2018). O estudo apresentado no artigo A20 relata a análise de um banco de dados nacional da Dinamarca, no qual os farmacêuticos clínicos de diversos hospitais inserem os dados de suas análises e intervenções (KJELDSEN et al., 2014). No artigo A19, pacientes em uso de medicamentos nefrotóxicos eram monitorizados e, ao sinal de alteração do clearance de creatina, eram sugeridos ajustes ou alterações na terapia medicamentosa (GOLDSTEIN et al., 2013).

A análise de prescrição de medicamentos antiácidos foi realizada no estudo A21, demonstrando que a atuação do farmacêutico garantiu o uso racional de medicamentos e resultados econômicos favoráveis (LUO et al., 2017). O artigo A18 relata a quantificação do tempo médio dispendido pelo farmacêutico na realização de diversas atividades clínicas (STUCHBERY et al., 2010). A atividade do farmacêutico, vinculada à adequação da prescrição aos protocolos clínicos e diretrizes terapêuticas para pacientes com diabetes, foi apresentada no artigo A22 (KHALIL et al., 2018).

Nesta revisão observou-se que apenas $31 \%$ dos estudos incluídos apresentam, em seu texto, a informação de registro da atividade clínica do farmacêutico no prontuário do paciente. Uma hipótese para a hesitação do farmacêutico em relação ao ato de registrar (evoluir) em prontuário as atividades clínicas, referentes ao cuidado do paciente, pode estar relacionada à inexperiência deste profissional referente à elaboração do conteúdo de registro (LIMA et al., 2017; PULLINGER; FRANKLIN, 2010).

Recente estudo realizado com farmacêuticos em hospital do sul do Brasil, mostrou que o principal fator limitante do registro em prontuário é a inexperiência (LIMA et al., 2017). Em 2010, um estudo feito em um hospital de Londres destacou que os farmacêuticos reconheciam quando, como e o que documentar, porém não realizavam o registro em prontuário, pois consideravam necessária formação adicional sobre o tema (PULLINGER; FRANKLIN, 2010). Para que ocorra a consolidação desta atividade por parte da classe farmacêutica, é necessário que ela seja adotada, difundi- da e incentivada pelas instituições de saúde e que seja conteúdo abordado em instituições de ensino superior (FOX; PEDERSEN; GUMPPER, 2015; MACKINNON; MACKINNON, 2011).

Um estudo realizado em 2016 analisou a apropriação das diretrizes curriculares nacionais por cursos de Graduação em Farmácia do sul do Brasil. A pesquisa verificou como dava-se a interface assistência farmacêutica e o ensino para atuação no Sistema Único de Saúde (SUS) nas grades curriculares dos cursos de Farmácia de algumas instituições de Ensino Superior do sul do país. Foi observado que, embora sejam oferecidas disciplinas com embasamento teórico, ainda havia pouca inserção dos acadêmicos em serviços de saúde vinculados ao SUS (MONTEGUTI; DIEHL, 2016).

O estudo de Monteguti e Diehl (2016) foi realizado antes da publicação das novas Diretrizes Curriculares Nacionais (DCNs) do curso de Graduação em Farmácia. As DCNs têm o intuito de qualificar o currículo dos cursos de Graduação e formar profissionais com habilidades para atuar na área de Farmácia Clínica, e possuem o eixo "Cuidado na Saúde", que prevê que os egressos de Farmácia sejam capacitados para "intervir de forma resolutiva nos problemas de saúde do indivíduo, da família e da comunidade bem como para planejar, executar e acompanhar ações em saúde" (BRASIL, 2017).

O registro das informações referentes ao cuidado em saúde é contemplado nestas diretrizes (BRASIL, 2017). Certamente a consolidação das práticas clínicas no projeto pedagógico refletirá em mudanças positivas na postura do profissional farmacêutico, que terá uma formação mais abrangente e será incorporado aos serviços de saúde com preparo para realizar o registro de suas intervenções no prontuário do paciente (LIMA et al., 2017; MONTEGUTI; DIEHL, 2016; BRASIL, 2017).

Estudo realizado a partir de entrevistas com pacientes que possuíam registro farmacêutico no prontuário eletrônico de um hospital de uma província canadense, apresentou que aproximadamente $50 \%$ dos pacientes que receberam cuidados deste profissional recordaram da intervenção, mostraram-se satisfeitos com o cuidado e relataram que gostariam de receber serviços farmacêuticos hospitalares se necessária nova internação (DOUCETTE et al., 2013).

$\mathrm{O}$ ato de registrar a atividade clínica em prontuário, além de ser um dever do profissional farmacêutico, é uma forma de evidenciar em documento oficial de valor legal a assistência prestada ao paciente (BRASIL, 2011). O prontuário é o documento definitivo do cuidado do paciente, e deve sempre ser o ponto de referência principal para o registro (PSNZ, 2014). Embora 
hajam recomendações referentes à necessidade do registro das atividades clínicas em prontuário, ainda não há diretrizes que norteiem quais informações e intervenções e como deve ser o registro do farmacêutico em prontuário (ADAM et al., 2019; BARANSKI et al., 2017).

É necessário compreender que o registro auxilia no processo, transição e continuidade do cuidado. Além de ser uma excelente ferramenta de comunicação entre a equipe multidisciplinar e valorosa fonte de dados para estudos, a documentação é essencial para avaliar o impacto global de um serviço (BRASIL, 2011; APHA, 2007).

\section{CONCLUSÃO}

Esta revisão integrativa demonstrou que uma pequena parcela dos artigos sobre atividades clínicas do farmacêutico em hospital, deixa claro que o registro referente à prática de cuidado ao paciente foi realizado em prontuário. A maioria dos artigos relata o registro das atividades clínicas em um banco de dados distinto e, mesmo os artigos que trazem a informação do registro em prontuário, também relatam a documentação da atividade em algum outro instrumento.

O fato de o farmacêutico inserir informações referentes a suas atividades clínicas em bases de dados distinta do prontuário, a fim de sistematizá-los para consultas futuras, pode facilitar a realização de pesquisas e mensuração de desfechos clínicos e econômicos. $\mathrm{O}$ ato de registrar uma ação clínica no prontuário do paciente, contudo, não pode ser negligenciado, pois é um direito do paciente e um dever do profissional de saúde.

Além do exposto previamente, o registro em prontuário garante a continuidade do cuidado pela equipe de farmácia e a manutenção e acesso à informação por parte de toda a equipe de saúde, aprimorando a comunicação e refletindo positivamente nos resultados dos pacientes. $O$ ato de documentar valida a responsabilidade e valoriza os serviços do profissional farmacêutico.

Esta revisão integrativa tem como principal limitação a possibilidade de não inclusão de estudos que contemplariam o tema em análise e os critérios de inclusão, porém não estão publicados em revistas indexadas nas bases de informação selecionadas para este estudo.

\section{REFERÊNCIAS}

ADAM, J.-P. et al. Documentation in the Patient's Medical Record by Clinical Pharmacists in a Canadian University Teaching Hospital. The Canadian journal of hospital pharmacy, v. 72, n. 3, p. 194-201, May./June 2019.
ALSULTAN, M. S. et al. Hospital pharmacy practice in Saudi Arabia: Drug monitoring and patient education in the Riyadh region. Saudi Pharmaceutical Journal, v. 21, n. 4, p. 361370, 2013.

APHA. American Pharmacists Association. Documenting Pharmacy-Based Patient Care Services, Module 5. Medication Therapy Management Services. Professional education monograph series for pharmacists from APhA. American Pharmacists Association, U.S.A., 2007.

ANDRÉS-LÁZARO, A. M. et al. Adecuación de la historia farmacoterapéutica y errores de conciliación en un servicio de urgencias. Medicina Clínica, v. 145, n. 7, p. 288-293, 2015.

BARANSKI, B. et al. Development of a Documentation Rubric and Assessment of Pharmacists' Competency for Documentation in the Patient Health Record. The Canadian Journal of Hospital Pharmacy, v. 70, n. 6, p. 423-429, Nov./ Dec., 2017.

BERNABÉ, E. M. et al. Sistemas de soporte a la toma de decisiones clínicas en insuficiencia renal. Farmacia Hospitalaria, v. 38, n. 3, p. 216-222, 2014.

BOTELHO, L. L. R.; CUNHA, C. C. D. A.; MACEDO, M. O método da revisão integrativa nos estudos organizacionais. Gestão e Sociedade, v. 5, n. 11, p. 121-136, 2011.

BOURNE, R. S.; CHOO, C. L. Pharmacist proactive medication recommendations using electronic documentation in a UK general critical care unit. International Journal of Clinical Pharmacy, v. 34, n. 2, p. 351-357, 2012.

BRASIL. Conselho Federal de Farmácia (CFF). Resolução no 476, de 28 de maio de 2008. Regulamenta o registro, a guarda e o manuseio de informações resultantes da prática da assistência farmacêutica nos serviços de saúde. Diário Oficial da União, Brasília, DF, 2008.

BRASIL. Conselho Federal de Farmácia (CFF). Resolução № 555, de 30 de novembro de 2011. Regulamenta o registro, a guarda e o manuseio de informações resultantes da prática da assistência farmacêutica nos serviços de saúde. Diário Oficial da União, Brasília, DF, Seção 1, p. 188, 14 dez. 2011.

BRASIL. Conselho Federal de Farmácia (CFF). Resolução no 585. Regulamenta as atribuições clínicas do farmacêutico e dá outras providências. Diário Oficial da União, Brasília, DF, Seção 1, p. 186, 25 set. 2013.

BRASIL. Ministério da Educação. Conselho Nacional de Educação. Câmara de Educação Superior. Resolução CNE/CES no 6, de 19 de outubro de 2017. Diretrizes Curriculares Nacionais do Curso de Graduação em Farmácia. Diário Oficial da União, Brasília, DF, Seção 1, p. 30, 20 out. 2017.

CUNHA, C.; BRANDÃO, A. Farmácia clínica: sonho, realização e história. Pharmacia Brasileira, p. 15-18, maio/jun. 2010.

DI PENTIMA, M. C.; CHAN, S. Impact of antimicrobial stewardship program on vancomycin use in a pediatric teaching hospital. The Pediatric Infectious Disease Journal, v. 29, n. 8, p. 707-711, 2010. 
DOUCETTE, D. et al. Patients' recall of interaction with a pharmacist during hospital admission. The Canadian Journal of Hospital Pharmacy, v. 66, n. 3, p. 171-176, May./June 2013.

DUNN, K. et al. Implementing a pharmacist-led sequential antimicrobial therapy strategy: a controlled before-and-after study. International Journal of Clinical Pharmacy, v. 33, p. 6, 2011.

ENGLISH, D.; ANKEM, K.; ENGLISH, K. Acceptance of clinical decision support surveillance technology in the clinical pharmacy. Informatics for Health and Social Care, v. 42, n. 2, p. 135-152, 2017.

FERRACINI, F. T. et al. Implantação e evolução da farmácia clínica no uso racional de medicamentos em hospital terciário de grande porte. einstein, v. 9, n. 4, Pt 1, p. 456-460, 2011.

FOX, B. I.; PEDERSEN, C. A.; GUMPPER, K. F. ASHP national survey on informatics: assessment of the adoption and use of pharmacy informatics in U.S. hospitals-2013. American Journal of Health-System Pharmacy, v. 72, n. 8, p. 636-655, 2015.

FRONTINI, R.; MIHARIJA-GALA, T.; SYKORA, J. EAHP survey 2010 on hospital pharmacy in Europe: parts 4 and 5. Clinical services and patient safety. European Journal of Hospital Pharmacy-Science and Practice, v. 20, n. 2, p. 69-73, Apr. 2013.

GARDNER, B.; GRANER, K. Pharmacists' medication reconciliation-related clinical interventions in a children's hospital. The Joint Commission Journal on Quality and Patient Safety, v. 35, n. 5, p. 278-282, 2009.

GOLDSTEIN, S. L. et al. Electronic health record identification of nephrotoxin exposure and associated acute kidney injury. Pediatrics, v. 132, n. 3, p. e756-767, 2013.

HESELMANS, A. et al. Medication review by a clinical pharmacist at the transfer point from ICU to ward: a randomized controlled trial. Journal of Clinical Pharmacy and Therapeutics, v. 40, n. 5, p. 578-583, Oct. 2015.

JURADO, L. V.; STEELMAN, J. D. The Role of the Pharmacist in the Intensive Care Unit. Critical Care Nursing Quarterly, v. 36, n. 4, p. 407-414, 2013.

KHALIL, V.; LI, M.; HUA, Q. A. An audit of the pharmacological management of ischaemic stroke patients in a metropolitan Australian hospital. International Journal of Clinical Pharmacy, v. 37, n. 1, p. 8-11. 2015.

KHALIL, V. et al. Antidiabetics' usage in type 2 diabetes mellitus: Are prescribing guidelines adhered to? A single centre study. Diabetes \& Metabolic Syndrome: Clinical Research \& Reviews, v. 12, n. 5, p. 635-641, 2018.

KJELDSEN, L. J. et al. Characterization of drug-related problems identified by clinical pharmacy staff at Danish hospitals. International Journal of Clinical Pharmacy, v. 36, n. 4, p. 734-741, 2014.

LAZARYAN, M. et al. Pharmacist Remote Review of Medication Prescriptions for Appropriateness in Pediatric Intensive Care Unit. Frontiers in Pharmacology, v. 7, p. 243-243, 2016.
LIMA, É. D. et al. Farmácia clínica em ambiente hospitalar: enfoque no registro das atividades. Revista Brasileira de Farmácia Hospitalar e Serviços de Saúde, v. 8, n. 4, p. 18-24, out./dez. 2017.

LOMBARDI, N. F. et al. Analysis of the discrepancies identified during medication reconciliation on patient admission in cardiology units: a descriptive study. Revista Latino-Americana de Enfermagem, v. 24, p. e2760. 2016.

LUO, H. et al. Impact of clinical pharmacist interventions on inappropriate prophylactic acid suppressant use in hepatobiliary surgical patients undergoing elective operations. PLoS One, v. 12, n. 10, p. e0186302-e0186302, 2017.

MACKINNON, G. E.; MACKINNON, N. J. Documentation of Pharmacy Services. In: DIPIRO, J. T. et al. (org.). Pharmacotherapy: A Pathophysiologic Approach. 8. ed. New York, NY: McGraw-Hill, p. 13-14, 2011.

MELNYK, B. M.; FINEOUT-OVERHOLT, E. Chapter 1: Making the Case for Evidence-Based Practice and Cultivating. In: MELNYK, B. M.; FINEOUT-OVERHOLT, E. Evidence-based practice in Nursing \& Healthcare: a guide to best practice a guide to best practice. $2^{\text {nd }}$ ed. Philadelphia: Lippincott Williams \& Wilkins, 2011. p. 3-24.

MENEZES, É. B. B. D. Atenção farmacêutica em xeque. Pharmacia Brasileira, Ano III, n. 22, p. 28, set./out. 2000.

MONGARET, C. et al. Predictive factors for clinically significant pharmacist interventions at hospital admission. Medicine, v. 97, n. 9, p. e9865-e9865, 2018.

MONTEGUTI, B. R.; DIEHL, E. E. Pharmacy education in Southern Brazil: preparing pharmacists for the unified health system? Trabalho, Educação e Saúde, v. 14, n. 1, p. 77-95, 2016.

MORGAN, S. R. et al. Clinical pharmacy services in the emergency department. The American Journal of Emergency Medicine, v. 36, n. 10, p. 1.727-1.732, 2018.

MORIEL, M. C. et al. Prospective Study on Conciliation of Medication in Orthopaedic Patients. Farmacia Hospitalaria, v. 32, n. 2, p. 65-70, 2008.

O'SULLIVAN, D. et al. The impact of a structured pharmacist intervention on the appropriateness of prescribing in older hospitalized patients. Drugs Aging, v. 31, n. 6, p. 471-481, 2014.

OKUMURA, L. M.; SILVA, M. M. G.; VERONEZE, I. Effects of a bundled Antimicrobial Stewardship Program on mortality: a cohort study. The Brazilian Journal of Infectious Diseases, v. 19, n. 3, p. 246-252, 2015.

PARENTE, D. M.; MORTON, J. Role of the Pharmacist in Antimicrobial Stewardship. Medical Clinics of North America, v. 102, n. 5, p. 929-936, 2018.

PASQUALE, T. R. et al. Impact of an antimicrobial stewardship program on patients with acute bacterial skin and skin structure infections. American Journal of Health-System Pharmacy, v. 71, n. 13, p. 1.136-1.139, 2014.

PAVÃO, A. L. B. et al. Incidence of in-hospital adverse events in the state of Rio de Janeiro, Brazil: evaluation of patient medical record. Revista Brasileira de Epidemiologia, v. 14, n. 4, p. 651-661, 2011. 
PSNZ. Pharmaceutical Society of New Zealand. New Zealand National Pharmacist Services Framework. Pharmaceutical Society of New Zealand Inc., p. 1-24, 2014.

PREBIANCHI, H. B. Comunicação da equipe interdisciplinar na assistência aos pacientes cirúrgicos. Temas em Educação e Saúde, v. 13, n.1, jan./jun. 2017.

PULLINGER, W.; FRANKLIN, B. D. Pharmacists' documentation in patients' hospital health records: issues and educational implications. International Journal of Pharmacy Practice, v. 18, n. 2, p. 108-115, 2010.

QUENNERY, S. et al. Added value of pharmacist-acquired drug histories in an orthopaedic ward. Acta Clinica Belgica, v. 66, n. 3, p. 196-199, May./June 2011.

SCHNEIDER, P. J.; PEDERSEN, C. A.; SCHECKELHOFF, D. J. ASHP national survey of pharmacy practice in hospital settings: Dispensing and administration-2017. American Journal of Health-System Pharmacy, v. 75, n. 16, p. 1.203-1.226, 2018.

STOREY, D. F. et al. Implementation of an antimicrobial stewardship program on the medical-surgical service of a 100-bed community hospital. Antimicrobial Resistance and Infection Control, v. 1, p. 8, 2012.

STUCHBERY, P. et al. Clinical pharmacy workload in medical and surgical patients: effect of patient partition, disease complexity and major disease category. International Journal of Pharmacy Practice, v. 18, n. 3, p. 159-166, 2010.

VAN NIEKERK, A. C.; VENTER, D. J. L.; BOSCHMANS, S.-A. Implementation of intravenous to oral antibiotic switch therapy guidelines in the general medical wards of a tertiary-level hospital in South Africa. Journal of Antimicrobial Chemotherapy, v. 67, n. 3, p. 756-762, 2011.

WAITMAN, L. R. et al. Adopting Real-Time Surveillance Dashboards as a Component of an Enterprisewide Medication Safety Strategy. The Joint Commission Journal on Quality and Patient Safety, v. 37, n. 7, p. 326-AP4, 2011.

WHITNEY, L. et al. Effectiveness of an antifungal stewardship programme at a London teaching hospital 2010-16. Journal of Antimicrobial Chemotherapy, p. dky389-dky389, 2019.

ZHANG, H.-X. et al. Pharmacist interventions for prophylactic antibiotic use in urological inpatients undergoing clean or clean-contaminated operations in a Chinese hospital. PLoS One, v. 9, n. 2, p. e88971-e88971, 2014.

ZHOU, L. et al. Optimizing Prophylactic Antibiotic Practice for Cardiothoracic Surgery by Pharmacists' Effects. Medicine, v. 95, n. 9, p. e2753-e2753, 2016. 